\title{
Notes on the Analysis of Cast Bronze ${ }^{1,2}$
}

\author{
By G. E. F. Lundell and J. A. Scherrer
}

Bureau of Standards, Department of Commerce, Washington, D. C.

\begin{abstract}
Many of the observations which are noted in the following pages are known, but they merit repetition because they represent desirable procedures and precautions which are often ignored. The method of analysis which is given is based on work done at the Bureau of Standards and by the cooperating analysts. It embodies nothing that is new, but possesses merit in that it groups desirable procedures and takes into account the known sources of error.
\end{abstract}

$\mathbf{M}^{\circ}$ OST of the observations contained in this paper were made in connection with the analysis of the Bureau of Standards standard sample of Cast Bronze No. 52. This bronze was chill-cast in 1 in. $\times 12$ in. $\times 24$ in. slabs under the supervision of Mr. P. E. McKinney at the Naval Gun Factory, Washington, D. C., and subsequently machined and mixed at the Bureau. Preliminary analyses demonstrated that the mixed sample was remarkably uniform in composition and that it was free from arsenic and phosphorus. The general composition of the bronze was fixed by the Advisory Committee on Nonferrous Metals and the actual composition as determined at the Bureau and by the coöperating analysts is as follows: copper, 88.33; tin, 7.90 ; zinc, 1.89 ; lead, 1.52; antimony, 0.16; nickel, 0.13 ; and iron, 0.12 . The following firms coöperated with the Bureau of Standards in its analysis:

$\begin{array}{ll}\text { The American Brass Co. } & \text { The National Cash Register Co. } \\ \text { Bridgeport Brass Co. } & \text { Crane Company } \\ \text { Scovill Manufacturing Co. } & \text { Ledoux and Co. } \\ \text { The Ajax Metal Co. } & \text { A. D. Little, Inc. } \\ \text { National Lead Co. } & \text { Pennsylvania Railroad System } \\ \text { Lumen Bearing Co. } & \text { C. B. \& Q. Railroad Co. } \\ \text { Lunkenheimer Co. } & \text { Naval Gun Factory, Washington, D. C. }\end{array}$

NOTES ON THE METHODS OF ANALYSIS

SEPARATION OF TIN AND ANTIMONY BY DIGESTION WITH NITRIC ACID-In this universally employed separation, the following observations are worthy of note:

(1) Hot digestion of the nitric acid solution of the alloy for 3 to 6 hrs. is desirable and it is imperative to keep the solution and the washing solution boiling hot throughout the filtering operation.

(2) Evaporation of the nitric acid solution to dryness followed by drenching and digestion with nitric acid is not necessary with alloys containing only tin or ten times as much tin as antimony. The operation does no harm, however, and is desirable with alloys of high antimony content, although in this case complete deposition of antimony is impossible.

(3) Other elements like phosphorus, arsenic and vanadium are quantitatively carried down with tin, provided the ratio of tin to these elements is high enough; in addition, the tin precipitate always carries down more or less of such other alloy constituents as iron, copper, zinc, and silicon.

(4) The complete separation of tin and antimony is often a matter of doubt, owing to such factors as the presence of considerable iron (over 0.25 per cent), the presence of considerable antimony, too short a digestion period, or the acceptance of a filtrate which is not crystal-clear.

DETERMINATION OF COPPER-(1) Equally good determinations are obtained by slow electrolysis and by rapid electrolysis in a solenoid apparatus or with rotating cathodes.

(2) Traces of copper (0.1 to $0.3 \mathrm{mg}$.) escape deposition in carefully conducted electrolyses and no advantage is ordinarily gained by redeposition. In fact a mechanical loss may result, if the first deposit is dissolved without using proper precautions.

(3) If tin or antimony in the ionic state is present in the electrolyte, it will be deposited to some extent with the copper and consequently the deposit should be examined for these elements.

: Received February 3, 1922.

2 Published by permission of the Director of the Bureau of Standards.
DETERMINATION OF TIN-(1) Gravimetric determinations of tin as oxide average higher than determinations by other methods and require correction for antimony (and such other elements as phosphorus and arsenic) as separately determined, and for such contaminants as iron, copper, zinc, and silicon as obtained either by actual determination and deduction or by separation and reprecipitation of the tin and antimony.

(2) Stannic oxide and antimony tetroxide are very easily reduced and consequently all reducing gases must be very carefully excluded during the ignition, which is best performed in a porcelain crucible set in a hole in an asbestos shield. When so protected the oxides will stand the full blast.

(3) Ignited tin and antimony oxides can be conveniently put in solution by fusion in a platinum crucible (with occasional stirring with a short platinum rod) with fused borax followed by digestion of the cooled melt in warm, dilute hydrochloric acid. The solution can then be tested for impurities by treating the solution with an excess of potassium hydroxide and hydrogen sulfide.

(4) The electrolytic method affords erratic values, sometimes low, owing perhaps to the lack of a suitable test for complete deposition, and sometimes high, owing to inclusions; this latter effect is often corrected by "blank" electrolyses.

(5) The iodometric method yields excellent results, provided the iodine solution is standardized under like conditions against material of known tin content; the tin titer so obtained is higher than the theoretical. The method is also re markably free from interfering factors, the ordinary alloy constituents, as well as antimony and arsenic, being without effect.

DETERMINATION OF ANTIMONY-(1) Gravimetric determinations of antimony as $\mathrm{Sb}_{2} \mathrm{O}_{4}$ or $\mathrm{Sb}_{2} \mathrm{~S}_{3}$ tend to give high values, owing in part to the difficulty in completely removing contaminating impurities. As noted under the determination of tin, $\mathrm{Sb}_{2} \mathrm{O}_{4}$ is very easily reduced; $\mathrm{Sb}_{2} \mathrm{~S}_{3}$ cannot be satisfactorily ignited in paper.

(2) An accurate and desirable method is the modified Low procedure (oxidation of antimony from the trivalent to the quinquevalent condition by permanganate in cold hydrochloricsulfuric acid solution). The titer of the permanganate solution obtained by titration against a compound of known antimony content is higher than that obtained by calculation from the iron value. The only interfering substance encountered in ordinary materials is arsenic. Fortunately this is usually absent or present in such small amount that a satisfactory correction can be calculated from the amount present.

DETERMINATION OF LEAD-(1) The electrolytic method yields accurate results when but small amounts of lead are involved and when the electrolyte is free from such interfering substances as manganese and colloidal metastannic acid.

(2) The sulfate method is to be preferred when large amounts of lead are involved or when the electrolyte contains manganese or tin.

(3) There is no apparent advantage in precipitating lead as sulfate and then dissolving it in ammoniun acetate and reprecipitating as chromate or molybdate.

DETERMINATION OF zINC-(1) Equally good results are obtained by the precipitation of $\mathrm{ZnS}$ from formic acid or $0.01 \mathrm{~N}$ sulfuric acid solutions.

(2) The precipitation of zinc sulfide or phosphate is incomplete in the presence of glyoxime reagent.

(3) The precipitation of zinc as phosphate must be made with diammonium phosphate in very weak acetic acid solution, and the solution must be kept warm until the phosphate becomes crystalline.

(4) The conversion of zinc sulfide to sulfate requires care in order to drive off excess sulfuric acid without loss of sulfur trioxide from the sulfate.

(5) The ignition of zinc sulfide to zinc oxide must be carried out at a low initial temperature until carbon is destroyed, lest there be a reduction and volatilization of zinc; a final temperature of $850^{\circ} \mathrm{C}$. is required to convert completely to oxide the sulfate which is formed, and a temperature above $950^{\circ} \mathrm{C}$. must. be avoided on account of the volatility of zinc oxide. 
DETERMINATION OF IRON-(1) The greatest source of error lies in ignoring the iron which is always carried down by tin and antimony during a nitric acid attack; frequently all of the iron is carried down.

(2) In gravimetric determinations corrections should be applied for silica and the possibility of the presence of such elements as aluminium borne in mind.

(3) In volumetric work corrections as indicated in blank determinations should be applied. It is to be remembered that Jones reductors always give a greater blank in the first run after disuse for a short period, and that solutions which have been treated with hydrogen sulfide contain oxidizable polythionic acids which must be destroyed by permanganate before final reduction with zinc or stannous chloride.

DETERMINATION OF NICKEI-(1) For small amounts of nickel the glyoxime method is unquestionably the most desirable. The glyoxime should be added to the hot acid solution which is then made ammoniacal. This results in quicker precipitation with small amounts of nickel than is obtained by reversing the procedure and the precipitate is more easily filtered. Digestion of the warm solution for one to two hours before filtration is desirable and hot water is the most satisfactory wash.

(2) In case nickel dimethylglyoxime is ignited to the oxide, care must be taken to prevent sublimation of the undecomposed salt.

(3) The electrolytic method tends to give high values with small amounts of nickel, owing in part to solution of the anode and partial deposition of platinum on the cathode.

\section{Method of ANaLYsis}

The most attractive method for the analysis of this type of material lies in a nitric acid attack followed by digestion and filtration to separate tin and antimony from the other elements. It is apparent, from what has been said, that the nitric acid filtrate must be examined for tin and antimony, while the nitric acid precipitate must be worked over for such contaminating elements as iron, copper, and zinc.

The following method calls for a nitric acid attack on two samples of bronze. In one sample, tin and antimony are filtered off, washed slightly, reserved, and then combined with any tin or antimony recovered from the filtrate. The tin and antimony are then put into sulfuric acid solution, the antimony is determined by titration with permanganate in the presence of hydrochloric acid, and the tin afterwards determined iodometrically in the same solution after reduction with test lead in an atmosphere of carbon dioxide. In the second sample, tin and antimony are filtered off washed slightly, the filtrate is reserved, and then combined with a nitric acid solution of any contaminants recovered from the tin and antimony. Copper and lead are then determined electrolytically. Zinc is thrown out in the electrolyte as sulfide in $0.01 \mathrm{~N}$ sulfuric acid solution and ignited to oxide. Iron is then precipitated twice by ammonia and weighed as oxide. Finally nickel is thrown out and weighed as nickel dimethylglyoxime. It is thus apparent that the method calls for volumetric determinations of antimony and tin, the simultaneous electrodeposition of copper and lead, and gravimetric determinations of zinc, iron, and nickel. As is shown, the gravimetric determination of lead and volumetric determinations of zinc, iron, and nickel can be substituted without changing the main procedure.

Gravimetric methods for tin, antimony, and lead are not favored, in spite of the desirability of finally having in an analysis a compound which may be seen and tested. The gravimetric method for tin as $\mathrm{SnO}_{2}$ is not specified on account of the impure character of the precipitate and the necessity of attempting corrections which, in the hands of novices, usually introduce greater errors than would the impurities. The volumetric method for antimony is preferred, in spite of the small amount present, on account of its rapidity and an accuracy equal to if not greater than that obtainable by ordinary care in a gravimetric determination as $\mathrm{Sb}_{2} \mathrm{O}_{4}$ or $\mathrm{Sb}_{2} \mathrm{~S}_{3}$.
The electrodeposition of lead is preferred in the absence of manganese and with the small amounts of lead involved, on account of the ease and rapidity of the method; there is no reason why lead cannot be separated and weighed as $\mathrm{PbSO}_{4}$ prior to the electrodeposition of copper.

\section{Determination of Antimony and Tin}

Digest $3 \mathrm{~g}$. of the sample with 50 cc. of nitric acid (sp. gr. 1.2) until decomposition is complete, boil to expel oxides of nitrogen, dilute with $150 \mathrm{cc}$. of hot water, and digest for 3 or $4 \mathrm{hrs}$. on the steam bath. Filter, keeping the solution hot, through a filter of close texture, and wash a few times with hot dilute nitric acid ( 1 per cent by volume). Reserve the precipitate. Nearly neutralize the filtrate with ammonia, pour the solution while stirring into an excess of ammonium sulfide, and digest at the side of the steam bath for an hour. Filter, wash slightly with water containing a little ammonium sulfide, and discard the precipitate. Acidify the filtrate with hydrochloric acid added in slight excess and digest at the side of the steam bath until the sulfur and sulfides settle. Filter, wash the residue with acidulated hydrogen sulfide water, and combine the paper and its contents with the reserved precipitate. ${ }^{3}$

ANTIMONY-Place the papers and precipitates in a 500-cc. Erlenmeyer flask and add 25 cc. of nitric acid (sp. gr. 1.42), $5 \mathrm{~g}$. of ammonium persulfate, and $15 \mathrm{cc}$. of sulfuric acid (sp. gr. 1.84). Boil until strong fumes of sulfuric acid escape. If the solution is brown, cool, and add $5 \mathrm{cc}$. of nitric acid and $1 \mathrm{~g}$. of persulfate, and boil again until strong fumes appear. ${ }^{4} \mathrm{Cool}$, dilute to $40 \mathrm{cc}$, add 0.5 to $1.0 \mathrm{~g}$. of sodium sulfite to reduce all of the antimony to the trivalent state, and boil until sulfur dioxide is expelled. Add 200 cc. of distilled water, 20 cc. of hydrochloric acid (sp. gr. 1.19), . cool to about $10^{\circ} \mathrm{C}$., and titrate with a standard solution of permanganate $^{5}$ to a pink tint which persists for 10 sec. The titration should be corrected by a blank determination (usually amounting to about 0.06 to $0.08 \mathrm{cc}$. of $0.1 \mathrm{~N}$ permanganate solution) carried out on a filter paper carried through all of the steps of the determination. ${ }^{6}$

TIN-Pour the titrated solution into a 500-cc. Erlenmeyer flask, add $80 \mathrm{cc}$. of hydrochloric acid (sp. gr. 1.19), and insert a 3-hole stopper carrying gas inlet and outlet tubes, and having the third hole closed by a small stopper. It is advisable to use an air condenser about 15 to $20 \mathrm{~cm}$. in length in the

\footnotetext{
${ }^{3}$ In case the alloy does not contain much iron or antimony the above recovery may be safely omitted in routine analyses.

4 This method of attack was tused with success by the American Brass Company. At this Bureau the paper and precipitate are placed in a Kjeldahl flask, treated with 2 to $4 \mathrm{~g}$. potassium sulfate and $20 \mathrm{cc}$ of sulfuric acid, and heated as in a nitrogen determination to complete solution of the sample and the destruction of organic matter.

- The permanganate solution should contain approximately $3.16 \mathrm{~g}$. potassium permanganate per liter. The theoretical antimony titer (approximately 0.006 ) as calculated by standardization against oxalate may be used, although it is preferable when dealing with an alloy of high antimony content or in accurate work to standardize under like conditions against material of known antimony content.

3 This determination is subject to interference by arsenic and vanadium So far as is known to the authors, vanadium is not employed in this class of material. Its presence could be easily detected by a hydrogen peroxide test of the titrated solution and its interference avoided by precipitating tin and antimony by hydrogen sulfide in acid solution, filtering, and then putting the sulfies into sulfuric acid solution. As regards the interference of arsenic, its prior removal is attended by uncertainty and by danger of volatilizing tin and, particularly, antimony. Therefore with the small amounts ordinarily present $(0.00$ to 0.03 per cent), it is safer to titrate, calculate the apparent percentage of antimony, and then deduct 1.86 times the percentage of arsenic as separately determined. (The factor 1.86 takes into account the difference in the atomic weights of antimony and arsenic and also the excess of permanganate required to get an end-point with arsenic.) With larger amounts of arsenic a modification such as that of
} Stief, ThIs JournaL, 7 (1915), 211, is called for. 
gas outlet. Add 1 to 2 g. of test lead, ${ }^{7}$ start a slow current of carbon dioxide from a Kipp generator or a cylinder, heat gradually to boiling, and boil gently for about $40 \mathrm{~min}$. Finally cool in ice to about $10^{\circ} \mathrm{C}$., after increasing the current of carbon dioxide in order to prevent back pressure. This may be detected by placing a bubbling tube in the gas outlet. When the solution is cold, take out the stopper in the third hole, add $5 \mathrm{cc}$. of clear starch solution from a pipet, and insert the tip of a buret containing standard iodine solution, ${ }^{8}$ and titrate to a permanent blue tint. The titration should be corrected by a blank determination (usually amounting to 0.05 to $0.08 \mathrm{cc}$. of $0.1 \mathrm{~N}$ iodine solution) carried through the steps of the procedure.

\section{Determination of Copper, Lejad, Zinc, Iron, AND NICKEL}

Treat $5 \mathrm{~g}$. of the sample as for the determination of antimony and tin, reserve the nitric acid solution, and treat the sulfuric acid solution of tin, antimony, and impurities with $10 \mathrm{~g}$. of tartaric acid, and sufficient 10 per cent potassium hydroxide solution to neutralize the acid and redissolve the precipitate formed. ${ }^{9}$ Pass in a stream of hydrogen sulfide for 10 to $15 \mathrm{~min}$., dilute to 600 to 800 cc., and digest for 2 to $3 \mathrm{hrs}$. on the steam bath, or preferably let stand over night. Filter and wash slightly with warm 1 per cent sodium or potassium sulfide solution, being careful not to allow the precipitate to run dry lest iron sulfide be oxidized and lost. Rinse as much of the sulfides as possible from the paper into the original beaker, dissolve the sulfide adhering to the paper in a small amount of hot dilute nitric acid (1:1) and boil to complete solution of the sulfides. Combine the nitric acid recovery with the reserved nitric acid filtrate and proceed as below.

COPPER AND IEAD-Neutralize the nitric acid solution with ammonia, add 1 cc. of nitric acid (sp. gr. 1.42) for every 100 cc. of solution, and electrolyze, using a platinum gauze cathode and sand-blasted gauze anode in a beaker of tall form covered with split glasses. When deposition is complete as demonstrated by the ordinary tests, the cathode and anode should be thoroughly washed with water as the beaker is lowered. Reserve the electrolyte. Dry the cathode and anode in the usual manner, weigh, and calculate copper ${ }^{10}$ and lead. ${ }^{11}$

I Some analysts prefer reduction by metallic antimony, iron, nickel, etc. The authors have found test lead to be very satisfactory, as it does not reduce in cold solution and hence does not require removal.

The iodine solution is prepared by dissolving about $12.7 \mathrm{~g}$. of iodine in a solution of about $20 \mathrm{~g}$. of potassium iodide in a little water, diluting to one liter, and standardizing against pure tin by the method described. The starch solution is prepared by allowing taw starch to stand for 24 hrs. in very dilute hydrochloric acid, filtering, washing, and drying at $100^{\circ} \mathrm{C}$. for 3 hrs. One gram of the hydrolyzed starch is then made into a paste with cold water: poured into $100 \mathrm{cc}$. of boiling water, boiled for $3 \mathrm{~min}$, cooled, and allowed to settle.

- In case antimony is absent, sodium hydroxide may be substituted for potassium hydroxide.

10 If the material contains much iron or antimony, or the most accurate results are desired, the copper deposit must be examined and corrected for tin and antimony as follows: Dissolve the weighed deposit in nitric acid ( 1 : 1), add $0.01 \mathrm{~g}$. of iron as ferric nitrate, boil, and precipitate with an excess of ammonia. Filter, wash the precipitate with hot water, and dissolve it in a small amount of hot dilute hydrochloric acid $(1: 1)$. Four the solution, while stirring, into a moderate excess of potassium hydroxide solution (10 per cent), pass in a rapid stream of hydrogen sulfide, and digest for a few hours on the steam bath. Filter, wash the precipitate slightly with dilute potassium sulfide solution and render the clear filtrate faintly acid with dilute hydrochloric acid. Allow the solution to stand at the side of the steam bath until the precipitate settles, filter, wash the paper and precipitate thoroughly with acidulated hydrogen sulfide water, and dissolve as much of it as possible in a little hot dilute nitric acid $(1: 1)$, keeping the solution. Burn the paper at the lowest possible temperature in a tared crucible, add the reserved nitric acid solution, evaporate to dryness, and ignite at a red heat in an oxidizing atmosphere. Multiply by the factor 0.788 (which
zINC-Evaporate the electrolyte and washings to small volume, add $10 \mathrm{cc}$. of dilute sulfuric acid $(1: 1)$ and heat to the appearance of fumes of sulfuric acid. Cool, dilute, treat with hydrogen sulfide, filter off any precipitate, and wash with dilute sulfuric acid ( 1 per cent by volume) saturated with hydrogen sulfide. ${ }^{12}$ Boil off hydrogen sulfide, add 2 drops of methyl orange solution and neutralize with ammonium hydroxide. Finally, dilute to about $100 \mathrm{cc}$. for every $0.1 \mathrm{~g}$. zinc and make $0.01 N$ with sulfuric acid ( 1 cc. $N$ acid to every 100 cc. of solution). Precipitate zinc as sulfide in cold solution, using a rapid current of hydrogen sulfide. Filter, wash with cold water, reserve the filtrate, and ignite the wet filter and contents very slowly in an oxidizing atmosphere until all carbon is destroyed. Gradually increase the temperature to $850^{\circ}$ for complete decomposition of the zinc sulfate formed. Do not heat above $900^{\circ} \mathrm{C}$. Cool and weigh as $\mathrm{ZnO}{ }^{13}$

IRON-Boil the filtrate reserved from the zinc determination until hydrogen sulfide and sulfur are expelled, using a few crystals of ammonium persulfate toward the end. Add a few drops of methyl red and then dilute ammonium hydroxide $(1: 1)$ drop by drop until the color of the solution changes to a distinct yellow. Boil the solution for 1 to 2 min., filter at once without washing, and reserve the filtrate. Dissolve the precipitate in a small amount of hot dilute hydrochloric acid $(1: 1)$, reprecipitate with ammonia as before, filter, and wash slightly with hot ammonium chloride solution (2 per cent). Add this filtrate to the one already reserved. Ignite the wet paper and precipitate in a weighed platinum crucible in an oxidizing atmosphere, treat with a drop of dilute sulfuric acid (1:1), 1 to 5 cc. of hydrofluoric acid, evaporate to dryness, again ignite, and weigh as $\mathrm{Fe}_{2} \mathrm{O}_{3}{ }^{14}$

NICKEL-Aeidify the combined filtrates from the iron determination, evaporate to approximately $300 \mathrm{cc}$, and filter if not absolutely clear. Nearly neutralize with ammonia, warm, add an excess of a 1 per cent alcoholic solution of dimethylglyoxime, and make slightly ammoniacal. After warm digestion for about $1 \mathrm{hr}$., filter on a weighed Gooch crucible, wash with hot water, dry at $110^{\circ}$ to $120^{\circ}$ for about

serves in these small amounts for both tin and antimony) and deduct from the weight of the copper deposit.

11 The lead deposit may be contaminated by silica, tin, and manganese. The first two are not ordinarily serious; in the case of manganese or when much lead is present, it is safer to precipitate lead as the sulfate in the combined nitric acid solutions and then proceed with the electrolysis of copper.

12 In accurate analyses the sulfides must be ignited, copper and lead determined, and proper corrections made. In routine analyses, they would be disregarded except when appreciable in amount.

13 Instead of the prescribed gravimetric procedure, the washed zinc sulfide may, in routine analyses, be dissolved in hydrochloric acid and titrated under the necessary definite conditions with a ferrocyanide solution which has been standardized under the same conditions against material of known zinc content.

14 The weighed precipitate will contain all of any aluminium that may have been in the bronze. The residue may therefore be examined for aluminium by fusing it with a little sodium carbonate, dissolving the cooled melt by digestion in dilute hydrochloric acid, reducing the almost neutral solution with a few drops of ammonium bisulfite and ther testing with phenylhydrazine as in the method of Hess and Campbell, as modified by E. T. Allen [J.Am. Chem. Soc, 25 (1903), 421].

Instead of the prescribed procedure, the fnal ammonia precipitate may be dissolved, reduced, and titrated. The advantage of the gravimetric procedure lies in the opportunity afforded for investigation of the character of the ammonia precipitate.

If iron alone is to be determined in the alloy, dissolve the weighed sample in aqua regia, boil, add ammonia in excess, and filter off the iron, lead, tin, and antimony. Dissolve the precipitate in a little hot dilute sulfuric acid, filter off any lead sulfate, and treat the acid solution with hydrogen sulfide. Filter, evaporate the filtrate to copious fumes of sulfuric acid, cool, and dilute. Finally add permanganate solution to a pink tint and proceed with the reduction and titration. The above method does not provide a satisfactory separation of aluminium on account of the large excess of ammonia which is necessary in the initial treatment. 